\title{
Persistence in mean and extinction on stochastic competitive Gilpin-Ayala systems with regime switching
}

\author{
Xiuli He' , Lei Liu ${ }^{1,2^{*}}$ and Quanxin Zhu ${ }^{3}$
}

"Correspondence:

liulei_hust@hhu.edu.cn

${ }^{1}$ College of Science, Hohai

University, Nanjing, Jiangsu 210098, China

${ }^{2}$ School of Mathematics, Southeast University, Nanjing, Jiangsu 210096, China

Full list of author information is available at the end of the article

\begin{abstract}
We are interested in the persistence in mean and extinction for a stochastic competitive Gilpin-Ayala system with regime switching. Based on the stochastic LaSalle theorem and the space-decomposition method, we derive generalized sufficient criteria on persistence in mean and extinction. By constructing a novel Lyapunov function we establish sufficient criteria on partial persistence in mean and partial extinction for the system. Finally, we provide two examples to demonstrate the feasibility and validity of our proposed methods.
\end{abstract}

Keywords: Lotka-Volterra model; random environments; Brownian motions; Itô formula; persistence in mean; extinction

\section{Introduction}

Many species live in the same environment, share the basic necessities, and compete for the limited recourses. This is a common phenomenon in the ecosystem. One of the most famous models is the classical competitive Lotka-Volterra system

$$
\frac{d x_{i}}{d t}=x_{i}\left(b_{i}-\sum_{j=1}^{n} a_{i j} x_{j}\right), \quad i=1,2, \ldots, n,
$$

where $x_{i}(t)$ represents the population size of species $i$ at time $t$, the constant $b_{i}$ is the growth rate of species $i$, and $a_{i j}$ represents the effect of interspecific $(i \neq j)$ or intraspecific $(i=j)$ interaction. One disadvantage is that the rate of change in the density of each species is a linear function. It might not describe the interaction behaviors of cross terms. Later on, to make up for this disadvantage and get more accurate results, Gilpin and Ayala have made some improvements on (1) and proposed the Gilpin-Ayala system

$$
\frac{d x_{i}}{d t}=x_{i}\left(b_{i}-a_{i i} x_{i}^{\theta_{i}}-\sum_{j \neq i} a_{i j} x_{j}\right), \quad i=1,2, \ldots, n
$$

where $\theta_{i}$ is the parameter to modify the classical Lotka-Volterra model. It is a nonlinear function and can better simulate the rate of density change.

(c) The Author(s) 2017. This article is distributed under the terms of the Creative Commons Attribution 4.0 International License (http://creativecommons.org/licenses/by/4.0/), which permits unrestricted use, distribution, and reproduction in any medium, provided you give appropriate credit to the original author(s) and the source, provide a link to the Creative Commons license, and indicate if changes were made. 
Afterward, the facts have been noticed that the population systems are inevitably affected by the environmental noise. One important type is the telegraph noise, which can be molded as a switching between two or more environment regimes. It is influenced by food and environmental factors. The other type is the widely known white noise, which is simulated by Brownian motion. Perturbed by these two important environmental noises, system (2) becomes the stochastic competitive Gilpin-Ayala system with regime switching

$$
\begin{aligned}
d x_{i} & =x_{i}\left(b_{i}(r(t))-a_{i i}(r(t)) x_{i}^{\theta_{i}}-\sum_{j \neq i} a_{i j}(r(t)) x_{j}\right) d t+\sigma_{i}(r(t)) x_{i} d B_{i}(t), \\
i & =1,2, \ldots, n .
\end{aligned}
$$

The stochastic competitive population system has been studied extensively because of its universal existence and importance (see e.g. [1-4]). The extinction and persistence in mean are the important and interesting properties of population systems, which mean that the population system will die out or survive in the future respectively. Therefore it has attracted considerable attention (see e.g. [5-8]). Li and Mao have investigated the dynamic behavior of several stochastic competitive Lotka-Volterra systems based on some novel Lyapunov functions, and some sufficient conditions are obtained in $[4,6,7]$, which insure the stochastic permanence or extinction. More recently, some improved sufficient conditions on persistence in mean or extinction for stochastic competitive Lotka-Volterra systems are provided by Liu and Shen [8] based on the space decomposition method. More details can be referred to $[9,10]$, and [11].

However, the approaches proposed by these authors (see e.g. [12-14], and [15]) for Lotka-Volterra systems cannot be easily used in stochastic Gilpin-Ayala systems because of the nonlinear item. Meanwhile, the methods proposed in [16] and [17] cannot be readily applied to the asymptotic analysis owing to the existence of regime-switching mechanism, which can make the problem insolvable. Moreover, to the best of our knowledge, the stochastic competitive Gilpin-Ayala system with regime switching and its asymptotic behavior have not been studied yet. The nonlinear item and the regime-switching mechanism exist in the model simultaneously, which might make the problem more complicated (see e.g. [18, 19], and [17] for more details). Based on this motivation, our aim is to tackle this issue. We first establish the detailed criteria for whole persistence in mean and whole extinction. Then we investigate the asymptotic properties for the partial persistence in mean and partial extinction. Finally, we give two examples to verify the feasibility and validity of our proposed methods.

\section{Notation}

Throughout this paper, unless otherwise specified, let $\left(\Omega, \mathcal{F},\left\{\mathcal{F}_{t}\right\}_{t \geq 0}, \mathbb{P}\right)$ be a complete probability space with filtration $\left\{\mathcal{F}_{t}\right\}_{t \geq 0}$ satisfying the usual conditions (i.e. it is increasing and right continuous, and $\mathcal{F}_{0}$ contains all $\mathbb{P}$-null sets). Let $B(t)=\left(B_{t}^{1}, \ldots, B_{t}^{n}\right)$ be an $n$-dimensional Brownian motion defined on the probability space.

Let $\{r(t), t \geq 0\}$ be a right-continuous Markovian chain on the probability space taking values in $S=\{1,2, \ldots, \mathrm{N}\}$ with generator $\Gamma=\left(\gamma_{i j}\right)_{\mathrm{N} \times \mathrm{N}}$ given by

$$
P\{r(t+\Delta)=j \mid r(t)=i\}= \begin{cases}\gamma_{i j} \Delta+o(\Delta), & i \neq j, \\ 1+\gamma_{i i} \Delta+o(\Delta), & i=j,\end{cases}
$$


where $\Delta>0$. Here $\gamma_{i j}>0$ is the transition rate from $i$ to $j$, and $\gamma_{i i}=-\sum_{j \neq i} \gamma_{i j}<0$ if $i \neq j$. We assume that the Markovian chain $r(t)$ is independent of the Brownian motion $B(t)$ and the Markovian chain has a unique stationary distribution $\pi=\left(\pi_{1}, \pi_{2}, \ldots, \pi_{n}\right)$.

For convenience and simplicity, we define

$$
\hat{a}_{i j}=\min _{k \in S} a_{i j}(k), \quad \check{a}_{i j}=\max _{k \in S} a_{i j}(k), \quad M_{i j}=\frac{\check{a}_{i j}}{\hat{a}_{i j}}, \quad m_{i j}=\frac{\hat{a}_{i j}}{\check{a}_{j j}}, \quad i, j=1, \ldots, n .
$$

For the sake of discussion, the whole frame is based on several basic assumptions.

Assumption $1 \theta_{i} \geq 1, a_{i i}>0, a_{i j} \geq 0, i, j=1,2, \ldots, n, i \neq j$.

Assumption $2 \sum_{k \in S} \pi_{k}\left(b_{i}(k)-\frac{\sigma_{i}^{2}(k)}{2}\right)>0$.

Assumption $3 \sum_{k \in S} \pi_{k}\left(\left(b_{i}(k)-\frac{\sigma_{i}^{2}(k)}{2}\right)-\sum_{j \neq i} a_{i j}(k)\left(\frac{b_{j}(k)-\frac{\sigma_{j}^{2}(k)}{2}}{a_{j j}(k)}\right)^{\frac{1}{\theta_{j}}}\right)>0, \forall j \neq i$.

We are motivated in particular by the work of Mao [13] and obtain the existence of a global positive solution.

Lemma 2.1 ([20]) Under Assumption 1, for any given initial values $x_{0} \in R_{+}^{n}$ and $r_{0} \in S$, there are a unique positive solution $x\left(t, x_{0}, r_{0}\right)$ of system (3) and a constant $K_{p}>0$ such that

$$
\sup _{0 \leq t<+\infty} \sum_{i=1}^{n} E x_{i}^{p}\left(t, x_{0}, r_{0}\right) \leq K_{p}, \quad i=1,2, \ldots, n .
$$

In the following sections, let $x_{i}(t)=x_{i}\left(t, x_{0}, r_{0}\right)$ for simplicity.

\section{Persistence in mean}

In this section, we investigate the persistence in mean.

Definition 1 System (3) is called persistent in mean if there exist constants $\alpha_{i}>0, \beta_{i}>0$, and $\gamma_{i} \geq 1$ such that

$$
\limsup _{t \rightarrow \infty} \frac{1}{t} \int_{0}^{t} x_{i}^{\gamma_{i}}(s) d s \leq \alpha_{i}, \quad \liminf _{t \rightarrow \infty} \frac{1}{t} \int_{0}^{t} x_{i}^{\gamma_{i}}(s) d s \geq \beta_{i} \quad \text { a.s. } i=1,2, \ldots, n .
$$

Consider two auxiliary stochastic differential equations

$$
\begin{aligned}
& \left\{\begin{array}{l}
d y_{i}=y_{i}\left(b_{i}(r(t))-a_{i i}(r(t)) y_{i}^{\theta_{i}}\right) d t+\sigma_{i}(r(t)) y_{i} d B_{i}(t), \\
y_{i}(0)=x_{i}(0), \quad r(0)=r_{0}, \quad i=1,2, \ldots, n ;
\end{array}\right. \\
& \left\{\begin{array}{l}
d z_{i}=z_{i}\left(b_{i}(r(t))-a_{i i}(r(t)) z_{i}^{\theta_{i}}-\sum_{j \neq i} a_{i j}(r(t)) y_{j}\right) d t+\sigma_{i}(r(t)) z_{i} d B_{i}(t), \\
z_{i}(0)=x_{i}(0), \quad r(0)=r_{0}, \quad i=1,2, \ldots, n .
\end{array}\right.
\end{aligned}
$$

Then it follows from the comparison principle (see [21]) that

$$
z_{i}(t) \leq x_{i}(t) \leq y_{i}(t), \quad i=1, \ldots, n
$$


Lemma 3.1 Let Assumption 1 and the inequality $\sum_{k \in S} \pi_{k}\left(b_{i}(k)-\frac{\sigma_{i}^{2}(k)}{2}\right) \geq 0$ hold. Then we have

$$
\lim _{t \rightarrow \infty} \frac{\log y_{i}(t)}{t}=0, \quad i=1,2, \ldots, n \text {, a.s. }
$$

The proof is omitted here; see [7] for more details.

Lemma 3.2 Let Assumptions 1 and 3 and the inequality $\sum_{k \in S} \pi_{k}\left(b_{i}(k)-\frac{\sigma_{i}^{2}(k)}{2}\right) \geq 0$ hold. Then the solution of system (3) satisfies

$$
\lim _{t \rightarrow \infty} \frac{\log x_{i}(t)}{t}=0, \quad i=1,2, \ldots, n, \text { a.s. }
$$

Proof By Lemma 3.1 and (6) we have derived $\lim \sup _{t \rightarrow \infty} \frac{\log x_{i}(t)}{t} \leq 0, i=1,2, \ldots, n$, a.s. Thus we still need to prove that $\liminf _{t \rightarrow \infty} \frac{\log x_{i}(t)}{t} \geq 0, i=1,2, \ldots, n$, a.s. It suffices to show that

$$
\liminf _{t \rightarrow \infty} \frac{\log z_{i}(t)}{t} \geq 0, \quad i=1,2, \ldots, n, \text { a.s. }
$$

Applying Itô’s formula to $z_{i}^{\theta_{i}}(t)$, we can readily verify that

$$
\begin{aligned}
\frac{1}{z_{i}^{\theta_{i}}(t)}= & \frac{1}{x_{i}^{\theta_{i}}(0)} \exp \left\{\left(\frac{\sigma_{i}^{2}(r(t))}{2}-b_{i}(r(t))\right) \theta_{i} t+\theta_{i} \sum_{j \neq i} \int_{0}^{t} a_{i j}(r(t)) y_{j}(s) d s\right\} \\
& -\theta_{i} \sigma_{i}(r(t)) B_{i}(t)+a_{i i}(r(t)) \theta_{i} \int_{0}^{t} \exp \left\{\left(\frac{\sigma_{i}^{2}(r(t))}{2}-b_{i}(r(t))\right) \theta_{i}(t-s)\right. \\
& \left.-\theta_{i} \sigma_{i}(r(t))\left(B_{i}(t)-B_{i}(s)\right)+\theta_{i} \sum_{j \neq i} \int_{s}^{t} a_{i j}(r(t)) y_{j}(\tau) d \tau\right\} d s \\
:= & E_{i 1}+E_{i 2} .
\end{aligned}
$$

A simple computation from (4) shows that

$$
\begin{aligned}
& \int_{s}^{t} y_{i}^{\theta_{i}}(\tau) d \tau \\
& \quad=\frac{b_{i}(r(t))}{a_{i i}(r(t))}(t-s)+\frac{\sigma_{i}(r(t))}{a_{i i}(r(t))}\left(B_{i}(t)-B_{i}(s)\right)+\frac{1}{a_{i i}(r(t))}\left(\ln y_{i}(s)-\ln y_{i}(t)\right) \\
& \quad=: c_{i}(t-s)+m_{i}\left(B_{i}(t)-B_{i}(s)\right)+d_{i}\left(\log y_{i}(s)-\log y_{i}(t)\right), \quad i=1,2, \ldots, n .
\end{aligned}
$$

The Hölder inequality then gives

$$
\begin{aligned}
\int_{s}^{t} y_{i}(\tau) d \tau & \leq(t-s)^{1-\frac{1}{\theta_{i}}}\left(\int_{s}^{t} y_{i}^{\theta_{i}}(\tau) d \tau\right)^{\frac{1}{\theta_{i}}} \\
& =(t-s)^{1-\frac{1}{\theta_{i}}}\left(c_{i}(t-s)+m_{i}\left(B_{i}(t)-B_{i}(s)\right)+d_{i}\left(\log y_{i}(s)-\log y_{i}(t)\right)\right)^{\frac{1}{\theta_{i}}} \\
\quad i=1,2, \ldots, n &
\end{aligned}
$$


It follows from the inequality $(a+b+c)^{p} \leq 3^{(p-1) \vee 0}\left(a^{p}+b^{p}+c^{p}\right)$ that

$$
\begin{aligned}
& \int_{s}^{t} y_{i}(\tau) d \tau \\
& \quad \leq(t-s)^{1-\frac{1}{\theta_{i}}}\left(c_{i}^{\frac{1}{\theta_{i}}}(t-s)^{\frac{1}{\theta_{i}}}+m_{i}^{\frac{1}{\theta_{i}}}\left(B_{i}(t)-B_{i}(s)\right)^{\frac{1}{\theta_{i}}}+d_{i}^{\frac{1}{\theta_{i}}}\left(\log y_{i}(s)-\log y_{i}(t)\right)^{\frac{1}{\theta_{i}}}\right), \\
& \quad i=1,2, \ldots, n .
\end{aligned}
$$

For $i=1,2, \ldots, n$, we define

$$
\begin{aligned}
& B_{i}^{*}(t):=B_{i}(t)-\min _{0 \leq s \leq t} B_{i}(s), \\
& \xi_{i}(t):=\max _{0 \leq s \leq t} \log y_{i}(s)-\log y_{i}(t) .
\end{aligned}
$$

Substituting the previous inequality into (10) yields

$$
\begin{aligned}
E_{i 1} \leq & \frac{1}{x_{i}^{\theta_{i}}(0)} \exp \left\{\left(\frac{\sigma_{i}^{2}(r(t))}{2}-b_{i}(r(t))\right) \theta_{i} t-\theta_{i} \sigma_{i}(r(t)) B_{i}(t)\right. \\
& +\theta_{i} \sum_{j \neq i} a_{i j}(r(t))(t-s)^{1-\frac{1}{\theta_{j}}}\left(c_{j}^{\frac{1}{\theta_{j}}}(t-s)^{\frac{1}{\theta_{j}}}\right. \\
& \left.\left.+m_{j}^{\frac{1}{\theta_{j}}}\left(B_{j}(t)-\min _{0 \leq s \leq t} B_{j}(s)\right)^{\frac{1}{\theta_{j}}}+d_{j}^{\frac{1}{\theta_{j}}}\left(\max _{0 \leq s \leq t} \log y_{j}(s)-\log y_{j}(t)\right)^{\frac{1}{\theta_{j}}}\right)\right\} \\
\leq & \frac{1}{x_{i}^{\theta_{i}}(0)} \exp \left\{\left(\frac{\sigma_{i}^{2}(r(t))}{2}-b_{i}(r(t))\right) \theta_{i} t+\theta_{i} \sigma_{i}(r(t))\left(\max _{0 \leq s \leq t} B_{i}(s)-B_{i}(t)\right)\right. \\
& \left.+\theta_{i} \sum_{j \neq i} a_{i j}(r(t)) t^{1-\frac{1}{\theta_{j}}}\left(c_{j}^{\frac{1}{\theta_{j}}} t^{\frac{1}{\theta_{j}}}+m_{j}^{\frac{1}{\theta_{j}}}\left(B_{j}^{*}(t)\right)^{\frac{1}{\theta_{j}}}+d_{j}^{\frac{1}{\theta_{j}}}\left(\xi_{j}(t)\right)^{\frac{1}{\theta_{j}}}\right)\right\} \\
\leq & \frac{1}{x_{i}^{\theta_{i}}(0)} \exp \left\{\left(\frac{\sigma_{i}^{2}(r(t))}{2}-b_{i}(r(t))+\sum_{j \neq i} a_{i j}(r(t)) c_{j}^{\frac{1}{\theta_{j}}}\right) \theta_{i} t\right. \\
& +\theta_{i} \sigma_{i}(r(t))\left(\max _{0 \leq s \leq t} B_{i}(s)-B_{i}(t)\right) \\
& \left.+\theta_{i} \sum_{j \neq i} a_{i j}(r(t)) t^{1-\frac{1}{\theta_{j}}}\left(m_{j}^{\frac{1}{\theta_{j}}}\left(B_{j}^{*}(t)\right)^{\frac{1}{\theta_{j}}}+d_{j}^{\frac{1}{\theta_{j}}}\left(\xi_{j}(t)\right)^{\frac{1}{\theta_{j}}}\right)\right\}, \quad i=1,2, \ldots, n .
\end{aligned}
$$

In the same way, we get

$$
\begin{aligned}
E_{i 2} \leq & a_{i i}(r(t)) \theta_{i} \int_{0}^{t} \exp \left\{\left(\frac{\sigma_{i}^{2}(r(t))}{2}-b_{i}(r(t))\right) \theta_{i}(t-s)\right. \\
& -\theta_{i} \sigma_{i}(r(t))\left(B_{i}(t)-B_{i}(s)\right)+\theta_{i} \sum_{j \neq i} a_{i j}(r(t))(t-s)^{1-\frac{1}{\theta_{j}}}\left(c_{j}^{\frac{1}{\theta_{j}}}(t-s)^{\frac{1}{\theta_{j}}}\right. \\
& \left.\left.+m_{j}^{\frac{1}{\theta_{j}}}\left(B_{j}(t)-\min _{0 \leq s \leq t} B_{j}(s)\right)^{\frac{1}{\theta_{j}}}+d_{j}^{\frac{1}{\theta_{j}}}\left(\max _{0 \leq s \leq t} \log y_{j}(s)-\log y_{j}(t)\right)^{\frac{1}{\theta_{j}}}\right)\right\} d s
\end{aligned}
$$




$$
\begin{aligned}
\leq & a_{i i}(r(t)) \theta_{i} \int_{0}^{t} \exp \left\{\left(\frac{\sigma_{i}^{2}(r(t))}{2}-b_{i}(r(t))+\sum_{j \neq i} a_{i j}(r(t)) c_{j}^{\frac{1}{\theta_{j}}}\right) \theta_{i}(t-s)\right. \\
& +\theta_{i} \sigma_{i}(r(t))\left(\max _{0 \leq s \leq t} B_{i}(s)-B_{i}(t)\right) \\
& \left.+\theta_{i} \sum_{j \neq i} a_{i j}(r(t)) t^{1-\frac{1}{\theta_{j}}}\left(m_{j}^{\frac{1}{\theta_{j}}}\left(B_{j}^{*}(t)\right)^{\frac{1}{\theta_{j}}}+d_{j}^{\frac{1}{\theta_{j}}}\left(\xi_{j}(t)\right)^{\frac{1}{\theta_{j}}}\right)\right\} d s, \quad i=1,2, \ldots, n .
\end{aligned}
$$

Substituting this into (10) yields

$$
\begin{aligned}
\frac{1}{z_{i}^{\theta_{i}}(t)} \leq & \left\{\frac{1}{x_{i}^{\theta_{i}}(0)} \exp \left(\frac{\sigma_{i}^{2}(r(t))}{2}-b_{i}(r(t))+\sum_{j \neq i} a_{i j}(r(t)) c_{j}^{\frac{1}{\theta_{j}}}\right) \theta_{i} t\right. \\
& \left.+a_{i i}(r(t)) \theta_{i} \int_{0}^{t} \exp \left[\left(\frac{\sigma_{i}^{2}(r(t))}{2}-b_{i}(r(t))+\sum_{j \neq i} a_{i j}(r(t)) c_{j}^{\frac{1}{\theta_{j}}}\right) \theta_{i}(t-s)\right] d s\right\} \\
& \times \exp \left\{\theta_{i} \sigma_{i}(r(t))\left(\max _{0 \leq s \leq t} B_{i}(s)-B_{i}(t)\right)\right. \\
& \left.+\theta_{i} \sum_{j \neq i} a_{i j}(r(t)) t^{1-\frac{1}{\theta_{j}}}\left(m_{j}^{\frac{1}{\theta_{j}}}\left(B_{j}^{*}(t)\right)^{\frac{1}{\theta_{i}}}+d_{j}^{\frac{1}{\theta_{j}}}\left(\xi_{j}(t)\right)^{\frac{1}{\theta_{j}}}\right)\right\} \\
:= & \phi_{i}^{-1}(t) \exp \left\{\theta_{i} \sigma_{i}(r(t))\left(\max _{0 \leq s \leq t} B_{i}(s)-B_{i}(t)\right)\right. \\
& \left.+\theta_{i} \sum_{j \neq i} a_{i j}(r(t)) t^{1-\frac{1}{\theta_{j}}}\left(m_{j}^{\frac{1}{\theta_{j}}}\left(B_{j}^{*}(t)\right)^{\frac{1}{\theta_{j}}}+d_{j}^{\frac{1}{\theta_{j}}}\left(\xi_{j}(t)\right)^{\frac{1}{\theta_{j}}}\right)\right\}, \quad i=1,2, \ldots, n,
\end{aligned}
$$

where $\phi_{i}(t)$ satisfies the system

$$
\left\{\begin{array}{l}
\dot{\phi}_{i}(t)=\theta_{i} \phi_{i}\left(b_{i}(r(t))-\frac{\sigma_{i}^{2}(r(t))}{2}-\sum_{j \neq i} a_{i j}(r(t)) c_{j}^{\frac{1}{\theta_{j}}}-a_{i i}(r(t)) \phi_{i}(t)\right) \\
\phi_{i}(0)=x_{i}^{\theta_{i}}(0), \quad i=1,2, \ldots, n
\end{array}\right.
$$

It follows from (16) that

$$
\begin{aligned}
\frac{\log z_{i}(t)}{t} \geq & \frac{\log \phi_{i}(t)}{\theta_{i} t}-\frac{\max _{0 \leq s \leq t} B_{i}(s)-B_{i}(t)}{t} \sigma_{i}(r(t)) \\
& -\sum_{j \neq i}\left(\frac{m_{j} B_{j}^{*}(t)}{t}\right)^{\frac{1}{\theta_{j}}}-\sum_{j \neq i}\left(\frac{d_{j} \xi_{j}(t)}{t}\right)^{\frac{1}{\theta_{j}}}, \quad \text { a.s. } i=1,2, \ldots, n .
\end{aligned}
$$

The Brownian motion implies

$$
\begin{aligned}
& \lim _{t \rightarrow \infty} \frac{\max _{0 \leq s \leq t} B_{i}(s)-B_{i}(t)}{t}=0, \\
& \lim _{t \rightarrow \infty} \frac{B_{j}(t)-\min _{0 \leq s \leq t} B_{j}(s)}{t}=0, \quad i=1,2, \ldots, n .
\end{aligned}
$$

When $b_{i}(r(t))-\frac{\sigma_{i}^{2}(r(t))}{2}-\sum_{j \neq i} a_{i j}(r(t)) c_{j}^{\frac{1}{\theta_{j}}}>0$, from (16) we can easily see that

$$
\lim _{t \rightarrow \infty} \frac{\log \phi_{i}(t)}{t}=0, \quad \text { a.s. } i=1,2, \ldots, n \text {. }
$$


Note that Lemma 3.1 implies

$$
\lim _{t \rightarrow \infty} \frac{\max _{0 \leq s \leq t} \log y_{j}(s)-\log y_{j}(t)}{t}=0 \quad \text { a.s., } i=1,2, \ldots, n .
$$

From (17) along with (18)-(20) we can verify that

$$
\liminf _{t \rightarrow \infty} \frac{\log z_{i}(t)}{t} \geq 0, \quad \limsup _{t \rightarrow \infty} \frac{\log z_{i}(t)}{t} \leq 0 \quad \text { a.s., } i=1,2, \ldots, n,
$$

as desired.

Remark 1 The convergence of (8) plays a prominent role in analyzing the asymptotic behavior for stochastic population systems. The property for stochastic Lotka-Volterra systems was discussed in [7], and the property for stochastic Gilpin-Ayala systems was studied in [18] as well. We discuss the system with highly nonlinear item and regime switching. These two factors limit the effectiveness, so the approach used in [7] and [18] is not applicable to system (3). By utilizing some novel techniques the asymptotic behavior for stochastic Gilpin-Ayala systems with regime switching has been studied, which might have a wider application.

Theorem 3.1 Let system (3) satisfy the hypotheses of Assumptions 1-3. Then it is said to be persistent in mean, that is,

$$
\begin{aligned}
& \liminf _{t \rightarrow \infty} \frac{1}{t} \int_{0}^{t} x_{i}^{\theta_{i}}\left(s, x_{0}, r_{0}\right) d s \\
& \quad \geq \frac{1}{\check{a}_{i i}}\left(\sum_{k \in S} \pi_{k}\left(b_{i}(k)-\frac{\sigma_{i}^{2}(k)}{2}\right)-\sum_{j \neq i} \check{a}_{i j}\left(\frac{1}{\hat{a}_{j j}} \sum_{k \in S} \pi_{k}\left(b_{j}(k)-\frac{\sigma_{j}^{2}(k)}{2}\right)\right)^{\frac{1}{\theta_{j}}}\right), \\
& \limsup _{t \rightarrow \infty} \frac{1}{t} \int_{0}^{t} x_{i}^{\theta_{i}}\left(s, x_{0}, r_{0}\right) d s \leq \frac{1}{\hat{a}_{i i}} \sum_{k \in S} \pi_{k}\left(b_{i}(k)-\frac{\sigma_{i}^{2}(k)}{2}\right) \quad \text { a.s., } i=1,2, \ldots, n .
\end{aligned}
$$

Proof Our proof is adapted from the works [8] and [18]. It follows by Itô's formula that

$$
\begin{aligned}
\log y_{i}(t)= & \log y_{i}(0)+\int_{0}^{t}\left(b_{i}(r(s))-\frac{\sigma_{i}^{2}(r(s))}{2}\right) d s \\
& -\int_{0}^{t} a_{i i}(r(s)) y_{i}^{\theta_{i}}(s) d s+\int_{0}^{t} \sigma_{i}(r(s)) d B_{i}(s) \quad \text { a.s., } i=1,2, \ldots, n .
\end{aligned}
$$

Thus, it satisfies

$$
\begin{aligned}
\frac{1}{t} \int_{0}^{t} a_{i i}(r(s)) y_{i}^{\theta_{i}}(s) d s= & \frac{1}{t}\left(\log y_{i}(0)-\log y_{i}(t)\right) \\
& +\frac{1}{t} \int_{0}^{t}\left(b_{i}(r(s))-\frac{\sigma_{i}^{2}(r(s))}{2}\right) d s+\frac{1}{t} \int_{0}^{t} \sigma_{i}(r(s)) d B_{i}(s)
\end{aligned}
$$$$
\text { a.s., } i=1,2, \ldots, n \text {. }
$$ 
Note that, for $i=1,2, \ldots, n, \frac{1}{T} \int_{0}^{T} \sigma_{i}(r(s)) d s<\infty$. By the strong law of large numbers for martingales we get

$$
\begin{aligned}
& \lim _{t \rightarrow \infty} \frac{1}{t} \int_{0}^{t} \sigma_{i}(r(s)) d B_{i}(s)=0 \quad \text { a.s., } \\
& \lim _{t \rightarrow \infty} \frac{1}{t} \int_{0}^{t}\left(b_{i}(r(s))-\frac{\sigma_{i}^{2}(r(s))}{2}\right) d s=\sum_{k \in S} \pi_{k}\left(b_{i}(k)-\frac{\sigma_{i}^{2}(k)}{2}\right) \\
& \text { a.s., } i=1,2, \ldots, n .
\end{aligned}
$$

By Lemma 3.1 we obtain from (22) that

$$
\begin{aligned}
& \lim _{t \rightarrow \infty} \frac{1}{t} \int_{0}^{t} a_{i i}(r(s)) y_{i}^{\theta_{i}}(s) d s=\sum_{k \in S} \pi_{k}\left(b_{i}(k)-\frac{\sigma_{i}^{2}(k)}{2}\right) \\
& \text { a.s., } i=1,2, \ldots, n .
\end{aligned}
$$

Therefore, for $i=1,2, \ldots, n$, we have

$$
\begin{aligned}
\limsup _{t \rightarrow \infty} \frac{1}{t} \int_{0}^{t} x_{i}^{\theta_{i}}(s) d s & \leq \limsup _{t \rightarrow \infty} \frac{1}{t} \int_{0}^{t} y_{i}^{\theta_{i}}(s) d s \\
& \leq \frac{1}{\hat{a}_{i i}} \limsup _{t \rightarrow \infty} \frac{1}{t} \int_{0}^{t} a_{i i}(r(s)) y_{i}^{\theta_{i}}(s) d s \\
& =\frac{1}{\hat{a}_{i i}} \sum_{k \in S} \pi_{k}\left(b_{i}(k)-\frac{\sigma_{i}^{2}(k)}{2}\right) \text { a.s. }
\end{aligned}
$$

Applying Itô’s formula to $z_{i}^{\theta_{i}}(t)$ yields

$$
\begin{aligned}
\log z_{i}(t)= & \log z_{i}(0)+\int_{0}^{t}\left(b_{i}(r(s))-\frac{\sigma_{i}^{2}(r(s))}{2}\right) d s-\int_{0}^{t} a_{i i}(r(s)) z_{i}^{\theta_{i}}(s) d s \\
& -\int_{0}^{t} \sum_{j \neq i} a_{i j}(r(s)) y_{j}(s) d s+\int_{0}^{t} \sigma_{i}(r(s)) d B_{i}(s) \\
\text { a.s., } i= & 1,2, \ldots, n .
\end{aligned}
$$

The well-known Hölder inequality then gives

$$
\frac{1}{t} \int_{0}^{t} x_{i} d s \leq\left(\frac{1}{t} \int_{0}^{t} x_{i}^{\theta_{i}}(s)\right)^{\frac{1}{\theta_{i}}}, \quad i=1,2, \ldots, n .
$$

Using Lemma 3.2 along with the Hölder inequality and the ergodic property of $r(t)$, we have

$$
\begin{aligned}
& \frac{1}{t} \int_{0}^{t} a_{i i}(r(s)) z_{i}^{\theta_{i}}(s) d s \\
& \quad=\frac{1}{t}\left(\log z_{i}(0)-\log z_{i}(t)\right)+\frac{1}{t} \int_{0}^{t}\left(b_{i}(r(s))-\frac{\sigma_{i}^{2}(r(s))}{2}\right) d s
\end{aligned}
$$




$$
\begin{aligned}
& -\frac{1}{t} \int_{0}^{t} \sum_{j \neq i} a_{i j}(r(s)) y_{j}(s) d s+\frac{1}{t} \int_{0}^{t} \sigma_{i}(r(s)) d B_{i}(s) \\
\geq & \frac{1}{t}\left(\log z_{i}(0)-\log z_{i}(t)\right)+\frac{1}{t} \int_{0}^{t}\left(b_{i}(r(s))-\frac{\sigma_{i}^{2}(r(s))}{2}\right) d s \\
& -\sum_{j \neq i} \check{a}_{i j}\left(\frac{1}{t} \int_{0}^{t} y_{j}^{\theta_{j}}(s) d s\right)^{\frac{1}{\theta_{j}}}+\frac{1}{t} \int_{0}^{t} \sigma_{i}(r(s)) d B_{i}(s) \\
\geq & \sum_{k \in S} \pi_{k}\left(b_{i}(k)-\frac{\sigma_{i}^{2}(k)}{2}\right)-\sum_{j \neq i} \check{a}_{i j}\left(\frac{1}{\hat{a}_{j j}} \sum_{k \in S} \pi_{k}\left(b_{j}(k)-\frac{\sigma_{j}^{2}(k)}{2}\right)\right)^{\frac{1}{\theta_{j}}} .
\end{aligned}
$$

We therefore have

$$
\begin{aligned}
& \liminf _{t \rightarrow \infty} \frac{1}{t} \int_{0}^{t} x_{i}^{\theta_{i}}(s) d s \\
& \geq \liminf _{t \rightarrow \infty} \frac{1}{t} \int_{0}^{t} z_{i}^{\theta_{i}}(s) d s \geq \frac{1}{\check{a}_{i i}} \limsup _{t \rightarrow \infty} \frac{1}{t} \int_{0}^{t} a_{i i}(r(s)) z_{i}^{\theta_{i}}(s) d s \\
& \geq \frac{1}{\check{a}_{i i}}\left(\sum_{k \in S} \pi_{k}\left(b_{i}(k)-\frac{\sigma_{i}^{2}(k)}{2}\right)-\sum_{j \neq i} \check{a}_{i j}\left(\frac{1}{\hat{a}_{j j}} \sum_{k \in S} \pi_{k}\left(b_{j}(k)-\frac{\sigma_{j}^{2}(k)}{2}\right)\right)^{\frac{1}{\theta_{j}}}\right),
\end{aligned}
$$

as desired. For $i=1,2, \ldots, n$, system (3) is persistent in mean.

Remark 2 When $\theta_{i}=1, i=1,2, \ldots, n$, system (3) reduces to the stochastic competitive Lotka-Volterra systems with regime switching, the conditions on persistence in mean are in accordance with the results in [8].

\section{Extinction}

In this section, we verify that the system will become extinct if the noise is sufficiently large.

Theorem 4.1 Suppose that Assumption 1 holds and there exists an integer $m \leq n$ such that

$$
\begin{aligned}
& \sum_{k \in S} \pi_{k}\left(b_{i}(k)-\frac{\sigma_{i}^{2}(k)}{2}\right)<0, \quad i=1,2, \ldots, m, \\
& \sum_{k \in S} \pi_{k}\left(b_{i}(k)-\frac{\sigma_{i}^{2}(k)}{2}\right)=0, \quad i=m+1, \ldots, n .
\end{aligned}
$$

\section{Then we have}

(i) The previous $m$ species of system (3) are almost surely exponentially extinct with the exponential rate of the ith species $-\sum_{k \in S} \pi_{k}\left(\frac{\sigma_{i}^{2}(k)}{2}-b_{i}(k)\right)$, that is,

$$
\lim _{t \rightarrow \infty} \frac{\log x_{i}\left(t, x_{0}, r_{0}\right)}{t}=-\sum_{k \in S} \pi_{k}\left(\frac{\sigma_{i}^{2}(k)}{2}-b_{i}(k)\right) \quad \text { a.s. }
$$

(ii) The following $n-m$ species will extinct with zero exponential rate, that is,

$$
\lim _{t \rightarrow \infty} x\left(t, x_{0}, r_{0}\right)=0 \quad \text { a.s. }
$$


Proof We begin by proving the exponential extinction for the top $m$ species of system (3) when $\sum_{k \in S} \pi_{k}\left(b_{i}(k)-\frac{\sigma_{i}^{2}(k)}{2}\right)<0, i=1,2, \ldots, m$. The next goal is to show the zero exponential extinction for the bottom $n-m$ species when $\sum_{k \in S} \pi_{k}\left(b_{i}(k)-\frac{\sigma_{i}^{2}(k)}{2}\right)=0, i=m+1, \ldots, n$.

Step 1. We aim to prove assertion (28). By Itô's formula we get

$$
\begin{aligned}
\log x_{i}(t)= & \log x_{i}(0)+\int_{0}^{t}\left(b_{i}(r(s))-\frac{\sigma_{i}^{2}(r(s))}{2}\right) d s \\
& \quad-\int_{0}^{t}\left(a_{i i}(r(s)) x_{i}^{\theta_{i}}(s)+\sum_{j \neq i} a_{i j}(r(s)) x_{j}(s)\right) d s+M_{i}(t), \\
& i=1,2, \ldots, n
\end{aligned}
$$

where $M_{i}(t)=\int_{0}^{t} \sigma_{i}(r(s)) d B_{i}(s), i=1,2, \ldots, n$. Dividing both sides by $t$ yields

$$
\begin{aligned}
& \frac{\log x_{i}(t)}{t}= \frac{\log x_{i}(0)}{t}+\frac{1}{t} \int_{0}^{t}\left(b_{i}(r(s))-\frac{\sigma_{i}^{2}(r(s))}{2}\right) d s \\
&-\frac{1}{t} \int_{0}^{t}\left(a_{i i}(r(s)) x_{i}^{\theta_{i}}(s)+\sum_{j \neq i} a_{i j}(r(s)) x_{j}(s)\right) d s+\frac{M_{i}(t)}{t}, \\
& i=1,2, \ldots, n .
\end{aligned}
$$

By the strong law of large numbers for martingales (see [20]) we derive $\lim _{t \rightarrow \infty} \frac{1}{t} \times$ $\int_{0}^{t} \sigma_{i}(r(s)) d B_{i}(s)=0$ a.s., $i=1,2, \ldots, n$. For $i=1,2, \ldots, m$, letting $t \rightarrow \infty$ on both sides of (30), we have

$$
\limsup _{t \rightarrow \infty} \frac{\log x_{i}(t)}{t} \leq-\sum_{k \in S} \pi_{k}\left(\frac{\sigma_{i}^{2}(k)}{2}-b_{i}(k)\right)<0, \quad i=1,2, \ldots, m \text {, a.s. }
$$

By (31), for any $1 \leq i \leq k$ and $\varepsilon \in\left(0, \min _{1 \leq i \leq k} \sum_{k \in S} \pi_{k}\left(\frac{\sigma_{i}^{2}(k)}{2}-b_{i}(k)\right)\right)$, we can select a random variable $T(\varepsilon)$ such that

$$
x_{i}(t) \leq \exp \left(-\sum_{k \in S} \pi_{k}\left(\frac{\sigma_{i}^{2}(k)}{2}-b_{i}(k)\right) t+\varepsilon t\right), \quad \forall t>T(\varepsilon), i=1,2, \ldots, m \text {, a.s. }
$$

Thus it follows that

$$
x_{i}^{\theta_{i}}(t) \leq \exp \left(-\theta_{i} \sum_{k \in S} \pi_{k}\left(\frac{\sigma_{i}^{2}(k)}{2}-b_{i}(k)\right) t+\theta_{i} \varepsilon t\right), \quad \forall t>T(\varepsilon), i=1,2, \ldots, m, \text { a.s. }
$$

Then we can readily verify that

$$
\int_{0}^{\infty}\left(a_{i i}(r(s)) x_{i}^{\theta_{i}}(s)+\sum_{j \neq i} a_{i j}(r(s)) x_{j}(s)\right) d s<\infty, \quad i=1,2, \ldots, m, \text { a.s. }
$$

By (30) and (33) we have

$$
\lim _{t \rightarrow \infty} \frac{\log x_{i}(t)}{t}=-\sum_{k \in S} \pi_{k}\left(\frac{\sigma_{i}^{2}(k)}{2}-b_{i}(k)\right), \quad i=1,2, \ldots, m, \text { a.s. }
$$


Step 2. We only need to show assertion (29). Applying Itô's formula to $\log x_{i}^{\theta_{i}}(t)$, we get

$$
\begin{aligned}
\frac{\log x_{i}(t)}{t}= & \frac{\log x_{i}(0)}{t}-\frac{1}{t} \int_{0}^{t} a_{i i}(r(s)) x_{i}^{\theta_{i}}(s) d s \\
& -\frac{1}{t} \sum_{j=1, j \neq i}^{n} \int_{0}^{t} a_{i j}(r(s)) x_{i}(s) d s+\frac{1}{t} \int_{0}^{t} \sigma_{i} d B_{i}(s) .
\end{aligned}
$$

Based on the convergence of the integral $\int_{0}^{\infty} x_{i}(s) d s$, the sample space $\Omega$ can be decomposed into two mutually exclusive events

$$
G_{i 1}=\left\{\omega: \int_{0}^{\infty} x_{i}(s) d s<\infty\right\} \text { and } G_{i 2}=\left\{\omega: \int_{0}^{\infty} x_{i}(s) d s=\infty\right\} .
$$

Furthermore, we can also divide $\Omega$ into three mutually exclusive events

$$
\begin{aligned}
& \digamma_{i 1}=\left\{\omega: \lim \sup _{t \rightarrow \infty} x_{i}(t) \geq \lim \inf _{t \rightarrow \infty} x_{i}(t)=\gamma_{i}>0\right\}, \\
& \digamma_{i 2}=\left\{\omega: \lim \sup _{t \rightarrow \infty} x_{i}(t)>\lim \inf _{t \rightarrow \infty} x_{i}(t)=0\right\}, \quad \text { and } \\
& \digamma_{i 3}=\left\{\omega: \lim _{t \rightarrow \infty} x_{i}(t)=0\right\} .
\end{aligned}
$$

The proof of $\lim _{t \rightarrow \infty} x_{i}(t)=0$ a.s. is equivalent to showing that $G_{i 1} \subset \digamma_{i 3}$ and $G_{i 2} \subset \digamma_{i 3}$ a.s. The following is an outline of the proof.

First, using stochastic LaSalle methods proposed in [22], we prove that $G_{i 1} \subset \digamma_{i 3}$. Second, using the novel techniques, we show that $P\left(G_{i 2} \cap \digamma_{i 1}\right)=0$ and $P\left(G_{i 2} \cap \digamma_{i 2}\right)=0$, which means that $G_{i 2} \subset \digamma_{i 3}$ a.s. Now we map out our strategy.

Case 1: The continuity of $x_{i}(t)$ and definition of $G_{i n}$ imply that $P\left(G_{i 1} \cap \digamma_{i 1}\right)=0$. Now we prove it by a contradiction.

Now let us show that $G_{i 1} \subset \digamma_{i 3}$. Clearly, $x_{i}(t) \in C\left(R_{+}, R\right)$ a.s. It is easy to check from $G_{i 1}$ that $\liminf _{t \rightarrow \infty} x_{i}(t)=0$ a.s. Therefore, we obtain that $P\left(G_{i 1} \cap \digamma_{i 1}\right)=0$. The only thing that remains to show is $P\left(G_{i 1} \cap \digamma_{i 2}\right)=0$. If $P\left(G_{i 1} \cap \digamma_{i 2}\right)>0$, then there exists a real number $\varepsilon>0$ such that

$$
P\left(Q_{1} \cap G_{i 1}\right) \geq 2 \varepsilon,
$$

where $Q_{1}=\left\{\lim \sup _{t \rightarrow \infty} x_{i}(t)>2 \varepsilon\right\}$. Define the sequence of stopping times

$$
\begin{aligned}
& \tau_{1}=\inf \left\{t \geq 0: x_{i}(t) \geq 2 \varepsilon\right\}, \quad \tau_{2 k}=\inf \left\{t \geq \tau_{2 k-1}: x_{i}(t) \leq \varepsilon\right\} \\
& \tau_{2 k+1}=\inf \left\{t \geq \tau_{2 k}: x_{i}(t) \geq 2 \varepsilon\right\}, \quad k=1,2, \ldots
\end{aligned}
$$

We have $E\left(I_{G_{i 1}} \int_{0}^{\infty} x_{i}(s) d s\right)<\infty$ from $G_{i 1}$. Then we compute and rearrange

$$
\begin{aligned}
E\left(I_{G_{i 1}} \int_{0}^{\infty} x_{i}(s) d s\right) & \geq \sum_{k=1}^{\infty} E\left(I_{\left\{\tau_{2 k-1}<\infty, \tau_{2 k}<\infty\right\} \cap G_{i 1}} \int_{\tau_{2 k-1}}^{\tau_{2 k}} x_{i}(s) d s\right) \\
& \geq \varepsilon \sum_{k=1}^{\infty} E\left(I_{\left\{\tau_{2 k-1}<\infty\right\} \cap G_{i 1}}\left(\tau_{2 k}-\tau_{2 k-1}\right)\right),
\end{aligned}
$$


where $I_{A}$ is the indicator function. Since $\tau_{2 k}<\infty$ whenever $\tau_{2 k-1}<\infty$, by the above formula we have

$$
\varepsilon \sum_{k=1}^{\infty} E\left(I_{\left\{\tau_{2 k-1}<\infty\right\} \cap G_{i 1}}\left(\tau_{2 k-1}-\tau_{2 k}\right)\right)<\infty
$$

Integrating equation (3), we have

$$
\begin{aligned}
x_{i}(t)= & x_{i}(0)+\int_{0}^{t} \sigma_{i} x_{i}(s) d B_{i}(s) \\
& +\int_{0}^{t} x_{i}(s)\left(b_{i}(r(s))-a_{i i}(r(s)) x_{i}^{\theta_{i}}(s)-\sum_{j=1, j \neq i}^{n} a_{i j}(r(s)) x_{j}(s)\right) d s .
\end{aligned}
$$

Compute and rearrange

$$
\begin{aligned}
& E\left\{x_{i}^{2}(s) \cdot\left(b_{i}(r(s))-a_{i i}(r(s)) x_{i}^{\theta_{i}}(s)-\sum_{j=1, j \neq i}^{n} a_{i j}(r(s)) x_{j}(s)\right)^{2}\right\} \\
& \leq \frac{1}{2} E\left(x_{i}^{4}(s)\right)+\frac{1}{2} E\left(b_{i}(r(s))-a_{i i}(r(s)) x_{i}^{\theta_{i}}(s)-\sum_{j=1, j \neq i}^{n} a_{i j}(r(s)) x_{j}(s)\right)^{4} \\
& \leq \frac{1}{2} E\left(x_{i}^{4}(s)\right)+\frac{1}{2} E\left(b^{4}+a_{i i}^{4} x_{i}^{4 \theta_{i}}+\sum_{j=1, j \neq i}^{n} a_{i j}^{4} x_{j}^{4}+4 a_{i i}^{3} x_{i}^{3 \theta_{i}} \sum_{j=1, j \neq i}^{n} a_{i j} x_{j}+4 a_{i i} x_{i}^{\theta_{i}} \sum_{j=1, j \neq i}^{n} a_{i j}^{3} x_{j}^{3}\right. \\
&\left.+6 b^{2} a_{i i}^{2} x_{i}^{2 \theta_{i}}+6 b^{2} \sum_{j=1, j \neq i}^{n} a_{i j}^{2} x_{j}^{2}+6 a_{i i}^{2} x_{i}^{2 \theta_{i}} \sum_{j=1, j \neq i}^{n} a_{i j}^{2} x_{j}^{2}+12 b^{2} a_{i i} x_{i}^{\theta_{i}} \sum_{j=1, j \neq i}^{n} a_{i j} x_{j}\right) \\
& \leq \frac{1}{2} K_{4}+\frac{1}{2}\left(b_{i}^{4}+a_{i i}^{4} K_{4 \theta_{i}}+\sum_{j=1, j \neq i}^{n} a_{i j}^{4} K_{4}+4 a_{i i}^{3} K_{3 \theta_{i}} \sum_{j=1, j \neq i}^{n} a_{i j} K_{1}+4 a_{i i} K_{\theta_{i}} \sum_{j=1, j \neq i}^{n} a_{i j}^{3} K_{3}\right. \\
&\left.+6 b^{2} a_{i i}^{2} K_{2 \theta_{i}}+6 b^{2} \sum_{j=1, j \neq i}^{n} a_{i j}^{2} K_{2}+6 a_{i i}^{2} K_{2 \theta_{i}} \sum_{j=1, j \neq i}^{n} a_{i j}^{2} K_{2}+12 b^{2} a_{i i} K_{\theta_{i}} \sum_{j=1, j \neq i}^{n} a_{i j} K_{1}\right) \\
&= U_{i}^{2}
\end{aligned}
$$

and

$$
E\left(\sigma_{i}^{2} \cdot x_{i}^{2}(s)\right)=\sigma_{i}^{2} \cdot E\left(x_{i}^{2}(s)\right) \leq \sigma_{i}^{2} \cdot K_{2}=: V_{i}^{2},
$$

where $K_{1}, K_{2}, K_{3}, K_{4}$ and $K_{\theta_{i}}, K_{2 \theta_{i}}, K_{3 \theta_{i}}, K_{4 \theta_{i}}$ are defined in Lemma 2.1. By the BDG inequality (see [20]) and the Hölder inequality we compute

$$
\begin{aligned}
& E\left(I_{\left\{\tau_{2 k-1}<\infty\right\} \cap G_{i 1}} \sup _{0 \leq t \leq T}\left|x_{i}\left(\tau_{2 k-1}+t\right)-x_{i}\left(\tau_{2 k-1}\right)\right|^{2}\right) \\
& \leq 2 E\left\{I_{\left\{\tau_{2 k-1}<\infty\right\} \cap G_{i 1}} \sup _{0 \leq t \leq T} \mid \int_{\tau_{2 k-1}}^{\tau_{2 k-1}+t} x_{i}(s)\left(b_{i}(r(s))-a_{i i}(r(s)) x_{i}^{\theta_{i}}(s)\right.\right. \\
& \left.\left.-\sum_{j=1, j \neq i}^{n} a_{i j}(r(s)) x_{j}(s)\right)\left.d s\right|^{2}\right\}
\end{aligned}
$$




$$
\begin{aligned}
& +2 E\left(I_{\left\{\tau_{2 k-1}<\infty\right\} \cap G_{i 1}} \sup _{0 \leq t \leq T}\left|\int_{\tau_{2 k-1}}^{\tau_{2 k-1}+t}\left(\sigma_{i} \cdot x_{i}(s)\right) d B_{i}(s)\right|^{2}\right) \\
\leq & 2 T E\left\{I _ { \{ \tau _ { 2 k - 1 } < \infty \} \cap G _ { i 1 } } \int _ { \tau _ { 2 k - 1 } } ^ { \tau _ { 2 k - 1 } + T } x _ { i } ^ { 2 } ( s ) \left(b_{i}(r(s))-a_{i i}(r(s)) x_{i}^{\theta_{i}}(s)\right.\right. \\
& \left.\left.-\sum_{j=1, j \neq i}^{n} a_{i j}(r(s)) x_{j}(s)\right)^{2} d s\right\} \\
& +8 E\left(I_{\left\{\tau_{2 k-1}<\infty\right\} \cap G_{i 1}} \int_{\tau_{2 k-1}}^{\tau_{2 k-1}+T}\left(\sigma_{i}^{2} \cdot x_{i}^{2}(s)\right) d s\right) \\
\leq & 2 T\left(U_{i}^{2}+4 V_{i}^{2}\right) .
\end{aligned}
$$

Choosing $T=T(\varepsilon)>0$ sufficiently small for $2 T\left(U_{i}^{2}+4 V_{i}^{2}\right) \leq \varepsilon^{3}$, from (38) we have

$$
P\left(\left\{\tau_{2 k-1}<\infty\right\} \cap\left\{H_{k} \cap J_{i 1}\right\}\right) \leq \frac{2(T+4) T\left(U_{i}^{2}+V_{i}^{2}\right)}{\varepsilon^{2}} \leq \varepsilon
$$

where $H_{k}=\left\{\sup _{1 \leq t \leq T}\left|x_{i}\left(\tau_{2 k-1}+t\right)-x_{i}\left(\tau_{2 k-1}\right)\right| \geq \varepsilon\right\}, k=1,2, \ldots$ Noting that $\tau_{k}<\infty$ for $k=1,2, \ldots$ whenever $\omega \in Q_{1}$, we further compute

$$
\begin{aligned}
& P\left(\left\{\tau_{2 k-1}<\infty\right\} \cap\left\{H_{k}^{c} \cap G_{i 1}\right\}\right) \\
& \quad=P\left(\left\{\tau_{2 k-1}<\infty\right\} \cap G_{i 1}\right)-P\left(\left\{\tau_{2 k-1}<\infty\right\} \cap\left\{H_{k} \cap G_{i 1}\right\}\right) \\
& \quad \geq 2 \varepsilon-\varepsilon=\varepsilon .
\end{aligned}
$$

Note that if $\omega \in\left\{\tau_{2 k-1}<\infty\right\} \cap\left\{H_{k}^{c} \cap G_{i 1}\right\}$, then

$$
\tau_{2 k}(\omega)-\tau_{2 k-1}(\omega) \geq T
$$

We obtain from (36) and (40) that

$$
\begin{aligned}
\infty & >\varepsilon \sum_{k=1}^{\infty} E\left[I_{\left\{\tau_{2 k-1}<\infty\right\} \cap G_{i 1}}\left(\tau_{2 k}-\tau_{2 k-1}\right)\right] \\
& \geq \varepsilon \sum_{k=1}^{\infty} E\left[I_{\left\{\tau_{2 k-1}<\infty\right\} \cap\left\{H_{k}^{c} \cap G_{i 1}\right\}}\left(\tau_{2 k-1}-\tau_{2 k}\right)\right] \\
& \geq \varepsilon T \sum_{k=1}^{\infty} P\left(\left\{\tau_{2 k-1}<\infty\right\} \cap\left\{H_{k}^{c} \cap G_{i 1}\right\}\right) \\
& \geq \varepsilon T \sum_{k=1}^{\infty} \varepsilon=\infty,
\end{aligned}
$$

which is a contraction. So $P\left(G_{i 1} \cap \digamma_{i 2}\right)=0$ holds, and we derive that $G_{i 1} \subset \digamma_{i 3}$.

Case 2. It remains to prove $G_{i 2} \subset \digamma_{i 3}$ a.s. We need only to show that $P\left(G_{i 2} \cap \digamma_{i 1}\right)=0$ and $P\left(G_{i 2} \cap \digamma_{i 2}\right)=0$. We prove it by a contradiction. If $P\left(G_{i 2} \cap \digamma_{i 1}\right)>0$, then for any $\omega \in$ $G_{i 2} \cap \digamma_{i 1}, \epsilon_{0} \in\left(0, \frac{\gamma_{i}}{2}\right)$, there exists $T=\left(\epsilon_{0}, \omega\right)$ such that

$$
x_{i}(t)>\gamma_{i}-\epsilon_{0}>\frac{\gamma_{i}}{2}, \quad \forall t>T \text {, a.s. }
$$


Then it follows that

$$
\begin{aligned}
\frac{1}{t} \int_{0}^{t} x_{i}(s) d s & =\frac{1}{t} \int_{0}^{T} x_{i}(s) d s+\frac{1}{t} \int_{T}^{t} x_{i}(s) d s \\
& \geq \frac{1}{t} \int_{0}^{T} x_{i}(s) d s+\frac{t-T}{t} \frac{\gamma_{i}}{2} \quad \text { a.s. }
\end{aligned}
$$

Letting $t \rightarrow \infty$, we get

$$
\liminf _{t \rightarrow \infty} \frac{1}{t} \int_{0}^{t} x_{i}(s) d s>\frac{\gamma_{i}}{2}>0 \quad \text { a.s. }
$$

This implies

$$
\limsup _{t \rightarrow \infty} \frac{\log x_{i}(t)}{t} \leq-\sum_{j=1}^{n} a_{i j} \frac{\gamma_{i}}{2}<0 \quad \text { a.s., }
$$

which contradicts the definition of $J_{i 2}$ and $\digamma_{i 1}$. So $P\left(G_{i 2} \cap \digamma_{i 1}\right)=0$ must be established. We proceed to show that $P\left(G_{i 2} \cap \digamma_{i 2}\right)>0$ is false. We need several notations:

$$
\begin{aligned}
\Gamma_{t}^{\varepsilon}(i) & :=\left\{0 \leq s \leq t: x_{i}(s) \geq \varepsilon\right\}, \\
\delta_{t}^{\varepsilon}(i) & :=\frac{m\left(\Gamma_{t}^{\varepsilon}(i)\right)}{t}, \\
\delta^{\varepsilon}(i) & :=\liminf _{t \rightarrow \infty} \delta_{t}^{\varepsilon} i, \\
\Delta^{\varepsilon}(i) & :=\left\{\omega \in J_{i 2} \cap \digamma_{i 2}: \delta^{\varepsilon}(i)>0\right\},
\end{aligned}
$$

where $m\left(\Gamma_{t}^{\varepsilon}(i)\right)$ denotes the length of $\Gamma_{t}^{\varepsilon}(i)$. It is easy to see that $\Delta^{0}(i)=G_{i 2} \cap \digamma_{i 2}$. Note that, for any $\varepsilon_{1}<\varepsilon_{2}$,

$$
\begin{aligned}
& \Gamma_{t}^{\varepsilon_{1}}(i) \supset \Gamma_{t}^{\varepsilon_{2}}(i), \quad m\left(\Gamma_{t}^{\varepsilon_{1}}(i)\right) \geq m\left(\Gamma_{t}^{\varepsilon_{2}}(i)\right), \\
& \delta_{t}^{\varepsilon_{1}}(i)=\frac{m\left(\Gamma_{t}^{\varepsilon_{1}}\right)(i)}{t} \geq \delta_{t}^{\varepsilon_{2}}(i)=\frac{m\left(\Gamma_{t}^{\varepsilon_{2}}\right)(i)}{t},
\end{aligned}
$$

which implies

$$
\delta^{\varepsilon_{2}}(i) \leq \delta^{\varepsilon_{1}}(i), \quad \Delta^{\varepsilon_{2}}(i) \subset \Delta^{\varepsilon_{1}}(i), \quad \forall \varepsilon_{1}<\varepsilon_{2} .
$$

By the continuity of probability we have

$$
P\left(\Delta^{\varepsilon}(i)\right) \rightarrow P\left(\Delta^{0}(i)\right)=P\left(G_{2} \cap \digamma_{2}\right) \quad \text { as } \varepsilon \rightarrow 0 .
$$

If $P\left(G_{i 2} \cap \digamma_{i 2}\right)>0$, then there exists $\varepsilon>0$ such that $P\left(D^{\varepsilon}\right)>0$. For any $\omega \in \Delta^{\varepsilon}(i)$, we have

$$
\begin{aligned}
\frac{1}{t} \int_{0}^{t} x_{i}(s) d s & =\frac{1}{t} \int_{\Pi_{t}^{\varepsilon}(i)} x_{i}(s) d s+\frac{1}{t} \int_{[0, t] \backslash \Pi_{t}^{\varepsilon}} x_{i}(s) d s \\
& \geq \frac{1}{t} \int_{\Pi_{t}^{\varepsilon}(i)} x_{i}(s) d s \quad \text { a.s. }
\end{aligned}
$$


Letting $t \rightarrow \infty$, we get

$$
\liminf _{t \rightarrow \infty} \frac{1}{t} \int_{0}^{t} x_{i}(s) d s \geq \liminf _{t \rightarrow \infty} \frac{1}{t} \int_{\Pi_{t}^{\varepsilon}} x_{i}(s) d s \geq \delta^{\varepsilon}(i) \varepsilon \quad \text { a.s. }
$$

Substituting (42) into (29), we have

$$
\limsup _{t \rightarrow \infty} \frac{\log x_{i}(t)}{t} \leq-\sum_{j=1}^{n} a_{i j} \delta^{\varepsilon}(i) \varepsilon<0 \quad \text { a.s. }
$$

This contradicts the definitions of $G_{i 2}$ and $\digamma_{i 2}$. Consequently, we conclude that $P\left(G_{i 2} \cap\right.$ $\left.\digamma_{i 2}\right)=0$. Combining the facts $G_{i 1} \subset \digamma_{i 3}, P\left(J_{i 2} \cap \digamma_{i 1}\right)=0$, and $P\left(G_{i 2} \cap \digamma_{i 2}\right)=0$, we derive

$$
\lim _{t \rightarrow \infty} x_{i}(t)=0 \quad \text { a.s., }
$$

as desired.

Remark 3 The difficulties come from the nonlinearities and regime switching. Based on stochastic LaSalle theorem and the space-decomposition method (see [8] and [22]), we overcome the difficulties. If system (3) does not contain parametric switching and $\theta_{i}=1$, $i=1,2, \ldots, n$, it happens to be the result in [7]. Therefore, Theorem 4.1 generalizes the results in [7] and [8].

\section{Partial persistence in mean and partial extinction}

We will discuss the partial persistence in mean and partial extinction on certain conditions later. Define the auxiliary stochastic differential equation:

$$
\left\{\begin{array}{l}
d u_{i}(t)=u_{i}\left(b_{i}(r(t))-a_{i i}(r(t)) u_{i}^{\theta_{i}}-\sum_{j=1, j \neq i}^{m} a_{i j}(r(t)) u_{j}\right) d t+\sigma_{i}(r(t)) d B_{i}(t) \\
u_{i}(0)=x_{i}(0), \quad r(0)=r_{0}, \quad i=1,2, \ldots, m
\end{array}\right.
$$

Theorem 5.1 Suppose that Assumption 1 holds and there exists an integer $1 \leq m \leq n$ such that

$$
\begin{aligned}
& \sum_{k \in S} \pi_{k}\left(b_{i}(k)-\frac{\sigma_{i}^{2}(k)}{2}\right)>0, \quad a_{i i}(k)-\sum_{j=1, j \neq i}^{m} a_{j i}(k)>0, \quad \forall k \in S, i=1,2, \ldots, m, \\
& \sum_{k \in S} \pi_{k}\left(\left(b_{i}(k)-\frac{\sigma_{i}^{2}(k)}{2}\right)-\sum_{j=1, j \neq i}^{m} \frac{a_{i j}(k)}{a_{j j}(k)}\left(b_{j}(k)-\frac{\sigma_{j}^{2}(k)}{2}\right)\right)>0, \\
& \quad i=1,2, \ldots, m, \\
& \sum_{k \in S} \pi_{k}\left(b_{i}(k)-\frac{\sigma_{i}^{2}(k)}{2}\right)<0, \quad i=m+1, \ldots, n .
\end{aligned}
$$


(i) For $i=1,2, \ldots, m$, the solution $x_{i}\left(t, x_{0}, r_{0}\right)$ to system (3) satisfies

$$
\begin{aligned}
& \liminf _{t \rightarrow \infty} \frac{1}{t} \int_{0}^{t} x_{i}^{\theta_{i}}(s) d s \\
& \geq \frac{1}{\check{a}_{i i}}\left\{\sum_{k \in S} \pi_{k}\left(b_{i}(k)-\frac{\sigma_{i}^{2}(k)}{2}\right)\right. \\
& \quad\left.-\sum_{j \neq i} \check{a}_{i j}\left(\frac{1}{\hat{a}_{j j}} \sum_{k \in S} \pi_{k}\left(b_{j}(k)-\frac{\sigma_{j}^{2}(k)}{2}\right)\right)^{\frac{1}{\theta_{j}}}\right\} \quad \text { a.s. } \\
& \limsup _{t \rightarrow \infty} \frac{1}{t} \int_{0}^{t} x_{i}^{\theta_{i}}(s) d s \leq \frac{1}{\hat{a}_{i i}} \sum_{k \in S} \pi_{k}\left(b_{i}(k)-\frac{\sigma_{i}^{2}(k)}{2}\right) \quad \text { a.s. }
\end{aligned}
$$

That is, the species $i$ is persistent in mean for each $i=1,2, \ldots, m$;

(ii) For $i=m+1, \ldots, n$, the solution $x_{i}\left(t, x_{0}, r_{0}\right)$ to system (3) satisfies

$$
\limsup _{t \rightarrow \infty} \frac{\log x_{i}(t)}{t} \leq \sum_{k \in S} \pi_{k}\left(b_{i}(k)-\frac{\sigma_{i}^{2}(k)}{2}\right) \quad \text { a.s. }
$$

that is, system (3) will become extinct.

Proof The proof is divided into two steps. The first step is to show the persistence in mean of the previous $m$ species. The second step is to show the extinction of the following $n-m$ species.

Step 1 . We analyze the convergence of $x_{i}^{\theta_{i}}(t)-u_{i}^{\theta_{i}}(t)$ as $t \rightarrow \infty$, where $u_{i}(t)$ is the solution to equation (43). Applying Itô's formula to system (43) yields

$$
d\left(\log u_{i}(t)\right)=\left(b_{i}(r(t))-a_{i i}(r(t)) u_{i}^{\theta_{i}}-\sum_{j=1, j \neq i}^{m} a_{i j}(r(t)) u_{j}\right) d t+\sigma_{i}(r(t)) d B_{i}(t)
$$

For $i=1,2, \ldots, m$, we have

$$
\begin{aligned}
& d\left(\log x_{i}(t)-\log u_{i}(t)\right) \\
& =-\sum_{j=1, j \neq i}^{m} a_{i j}(r(t))\left(x_{j}-u_{j}\right) d t-\sum_{j=m+1}^{n} a_{i j}(r(t)) x_{j} d t \\
& \quad-a_{i i}(r(t))\left(x_{i}^{\theta_{i}}-u_{i}^{\theta_{i}}\right) .
\end{aligned}
$$

Applying Itô's formula to $V(t)=\sum_{i=1}^{m}\left|\log x_{i}^{\theta_{i}}(t)-\log u_{i}^{\theta_{i}}(t)\right|$ yields

$$
\begin{aligned}
D^{+} V(t)= & \sum_{i=1}^{m} \operatorname{sign}\left(x_{i}^{\theta_{i}}(t)-u_{i}^{\theta_{i}}(t)\right)\left(d \log x_{i}^{\theta_{i}}(t)-d \log u_{i}^{\theta_{i}}(t)\right) \\
= & -\sum_{i=1}^{m} \operatorname{sign}\left(x_{i}^{\theta_{i}}(t)-u_{i}^{\theta_{i}}(t)\right) \\
& \cdot\left(\sum_{j=1, j \neq i}^{m} a_{i j}(r(t))\left(x_{j}^{\theta_{i}}-u_{j}^{\theta_{i}}\right) d t+\sum_{j=m+1}^{n} a_{i j}(r(t)) x_{j}^{\theta_{i}} d t+a_{i i}(r(t))\left(x_{i}^{\theta_{i}}-u_{i}^{\theta_{i}}\right)\right)
\end{aligned}
$$




$$
\begin{aligned}
\leq & -\sum_{i=1}^{m} a_{i i}(r(t))\left|x_{i}^{\theta_{i}}-u_{i}^{\theta_{i}}\right| d t+\sum_{i=1}^{m} \sum_{j=1, j \neq i}^{m} a_{i j}(r(t))\left|x_{j}^{\theta_{i}}-u_{j}^{\theta_{i}}\right| d t \\
& +\sum_{i=1}^{m} \sum_{j=m+1}^{n} a_{i j}(r(t)) x_{j}^{\theta_{i}} d t \\
= & -\sum_{i=1}^{m} a_{i i}(r(t))\left|x_{i}^{\theta_{i}}-u_{i}^{\theta_{i}}\right| d t+\sum_{j=1}^{m} \sum_{i=1, i \neq j}^{m} a_{j i}(r(t))\left|x_{i}^{\theta_{i}}-u_{i}^{\theta_{i}}\right| d t \\
& +\sum_{i=1}^{m} \sum_{j=m+1}^{n} a_{i j}(r(t)) x_{j}^{\theta_{i}} d t \\
\leq & -\sum_{i=1}^{m} a_{i i}(r(t))\left|x_{i}^{\theta_{i}}-u_{i}^{\theta_{i}}\right| d t+\sum_{i=1}^{m} \sum_{j=1, j \neq i}^{m} a_{j i}(r(t))\left|x_{i}^{\theta_{i}}-u_{i}^{\theta_{i}}\right| d t \\
& +\sum_{i=1}^{m} \sum_{j=m+1}^{n} a_{i j}(r(t)) x_{j}^{\theta_{i}} d t \\
= & -\sum_{i=1}^{m}\left(a_{i i}(r(t))-\sum_{j \neq i} a_{j i}(r(t))\right)\left|x_{i}^{\theta_{i}}(t)-u_{i}^{\theta_{i}}(t)\right| d t \\
& +\sum_{i=1}^{m} \sum_{j=m+1}^{n} a_{i j}(r(t)) x_{j}^{\theta_{i}}(t) d t .
\end{aligned}
$$

Then we have

$$
D^{+} V(t) \leq-\mu \sum_{i=1}^{m}\left|x_{i}^{\theta_{i}}(t)-u_{i}^{\theta_{i}}(t)\right| d t+\sum_{j=m+1}^{n} \alpha_{j} x_{j}^{\theta_{i}}(t) d t, \quad \text { a.s. } i=1,2, \ldots, m
$$

where $\mu=\min _{1 \leq i \leq m}\left(a_{i i}(r(t))-\sum_{j \neq i} a_{j i}(r(t))\right)>0$ and $\alpha_{j}=\sum_{i=1}^{m} a_{i j}(r(t)) \geq 0$. Therefore it satisfies

$$
\begin{aligned}
& V(t)+\mu \int_{0}^{t} \sum_{i=1}^{m}\left|x_{i}^{\theta_{i}}(s)-u_{i}^{\theta_{i}}(s)\right| d s \leq V(0)+\sum_{j=m+1}^{n} \alpha_{j} \int_{0}^{t} x_{j}^{\theta_{i}}(s) d s \\
& \text { a.s. } i=1,2, \ldots, m .
\end{aligned}
$$

Letting $t \rightarrow \infty$, we get

$$
\begin{aligned}
\int_{0}^{\infty}\left|x_{i}^{\theta_{i}}(s)-u_{i}^{\theta_{i}}(s)\right| d s & \leq \int_{0}^{\infty} \sum_{i=1}^{m}\left|x_{i}^{\theta_{i}}(s)-u_{i}^{\theta_{i}}(s)\right| d s \\
& \leq \frac{1}{\mu}\left(V(0)+\sum_{j=m+1}^{n} \alpha_{j} \int_{0}^{\infty} x_{j}^{\theta_{i}}(s) d s\right) .
\end{aligned}
$$

Note that by Theorem 4.1 and (45)

$$
\int_{0}^{\infty} x_{j}^{\theta_{i}}(s) d s<+\infty \quad \text { a.s. } j=m+1, \ldots, n .
$$


By (54), the inequality (53) satisfies

$$
\int_{0}^{\infty}\left|x_{i}^{\theta_{i}}(s)-u_{i}^{\theta_{i}}(s)\right| d s<+\infty \quad \text { a.s. } i=1,2, \ldots, m .
$$

Thus by Lemmas 5.1 and 5.2 (see [8] for more details) we have

$$
\lim _{t \rightarrow+\infty}\left(x_{i}^{\theta_{i}}(s)-u_{i}^{\theta_{i}}(s)\right)=0 \quad \text { a.s. } i=1,2, \ldots, m
$$

It follows from Theorem 3.1 that system (43) is persistent in mean, namely,

$$
\begin{aligned}
& \liminf _{t \rightarrow \infty} \frac{1}{t} \int_{0}^{t} u_{i}^{\theta_{i}}(s) d s \\
& \quad \geq \frac{1}{\check{a}_{i i}}\left\{\sum_{k \in S} \pi_{k}\left(b_{i}(k)-\frac{\sigma_{i}^{2}(k)}{2}\right)-\sum_{j \neq i} \check{a}_{i j}\left(\frac{1}{\hat{a}_{j j}} \sum_{k \in S} \pi_{k}\left(b_{j}(k)-\frac{\sigma_{j}^{2}(k)}{2}\right)\right)^{\frac{1}{\theta_{j}}}\right\} \\
& \limsup _{t \rightarrow \infty} \frac{1}{t} \int_{0}^{t} u_{i}^{\theta_{i}}(s) d s \leq \frac{1}{\hat{a}_{i i}} \sum_{k \in S} \pi_{k}\left(b_{i}(k)-\frac{\sigma_{i}^{2}(k)}{2}\right) \\
& \quad \text { a.s. } i=1,2, \ldots, n .
\end{aligned}
$$

For $i=1,2, \ldots, m$, note that (57) implies

$$
\begin{aligned}
& \liminf _{t \rightarrow \infty} \frac{1}{t} \int_{0}^{t} x_{i}^{\theta_{i}}(s) d s \\
& =\liminf _{t \rightarrow \infty} \frac{1}{t} \int_{0}^{t}\left(x_{i}^{\theta_{i}}(s)-u_{i}^{\theta_{i}}(s)\right) d s+\liminf _{t \rightarrow \infty} \frac{1}{t} \int_{0}^{t} u_{i}^{\theta_{i}}(s) d s \\
& \geq \frac{1}{\check{a}_{i i}}\left\{\sum_{k \in S} \pi_{k}\left(b_{i}(k)-\frac{\sigma_{i}^{2}(k)}{2}\right)-\sum_{j \neq i} \check{a}_{i j}\left(\frac{1}{\hat{a}_{j j}} \sum_{k \in S} \pi_{k}\left(b_{j}(k)-\frac{\sigma_{j}^{2}(k)}{2}\right)\right)^{\frac{1}{\theta_{j}}}\right\} \\
& \text { a.s. } i=1,2, \ldots, m \text {, } \\
& \limsup _{t \rightarrow \infty} \frac{1}{t} \int_{0}^{t} x_{i}^{\theta_{i}}(s) d s \\
& =\limsup _{t \rightarrow \infty} \frac{1}{t} \int_{0}^{t}\left(x_{i}^{\theta_{i}}(s)-u_{i}^{\theta_{i}}(s)\right) d s+\limsup _{t \rightarrow \infty} \frac{1}{t} \int_{0}^{t} u_{i}^{\theta_{i}}(s) d s \\
& \leq \frac{1}{\hat{a}_{i i}} \sum_{k \in S} \pi_{k}\left(b_{i}(k)-\frac{\sigma_{i}^{2}(k)}{2}\right) \quad \text { a.s., } i=1,2, \ldots, m \text {. }
\end{aligned}
$$

For $i=1,2, \ldots, m$, the solution $x_{i}(t)$ is persistent in mean.

Step 2 . For $i=m+1, \ldots, n$, applying Itô's formula to $\log x_{i}^{\theta_{i}}(t)$ yields

$$
\begin{aligned}
\frac{\log x_{i}(t)}{t}= & \frac{\log x(0)}{t}+\frac{1}{t} \int_{0}^{t}\left(b_{i}(r(s))-\frac{\sigma_{i}^{2}(r(s))}{2}\right) d s \\
& -\frac{1}{t} \int_{0}^{t} a_{i i}(r(s)) x_{i}^{\theta_{i}}(s) d s-\frac{1}{t} \int_{0}^{t} \sum_{j=1}^{m} a_{i j}(r(s)) x_{j}(s) d s+\frac{M_{i}(t)}{t} \\
& -\frac{1}{t} \int_{0}^{t} \sum_{j=m+1, j \neq i}^{n} a_{i j}(r(s)) x_{j}(s) d s \quad \text { a.s. } i=m+1, \ldots, n .
\end{aligned}
$$


It follows from Theorem 4.1 and (33) that

$$
\lim _{t \rightarrow \infty} \frac{1}{t} \int_{0}^{t} \sum_{j=m+1, j \neq i}^{n} a_{i j}(r(s)) x_{j}(s) d s=0 \quad \text { a.s. }
$$

By Theorem 3.1, we obtain that $x_{i}(t)$ is persistent in mean, which means that $\frac{1}{t} \times$ $\int_{0}^{t} \sum_{j=1}^{m} a_{i j}(r(s)) x_{j}(s) d s$ must be bounded. Letting $t \rightarrow \infty$ on both sides of (60), we have

$$
\limsup _{t \rightarrow \infty} \frac{\log x_{i}(t)}{t} \leq \sum_{k \in S} \pi_{k}\left(b_{i}(k)-\frac{\sigma_{i}^{2}(k)}{2}\right) \text { a.s. }
$$

as desired.

Remark 4 Compared to the whole persistence in mean and whole extinction, there is little literature on partial persistence in mean and partial extinction. By constructing a novel Lyapunov function we derive sufficient conditions for partial persistence in mean and partial extinction, which generalize the results in [8].

\section{Examples}

Example 6.1 Consider a two-dimensional stochastic Gilpin-Ayala system with regime switching in random environments

$$
\left\{\begin{array}{l}
d x_{1}=x_{1}\left(b_{1}(r(t))-0.6 x_{1}^{1.5}-0.5 x_{2}\right) d t+\sigma_{1}(r(t)) x_{1} d B_{1}(t), \\
d x_{2}=x_{2}\left(b_{2}(r(t))-0.3 x_{1}-x_{2}^{1.2}\right) d t+\sigma_{2}(r(t)) x_{2} d B_{2}(t)
\end{array}\right.
$$

where $r(t) \in\{1,2\}$ is generated by

$$
Q=\left(\begin{array}{cc}
-1 & 1 \\
\frac{1}{3} & -\frac{1}{3}
\end{array}\right)
$$

and $\pi=\left(\pi_{1}, \pi_{2}\right)=(0.25,0.75), b_{1}(1)=0.9, b_{1}(2)=1.2, b_{2}(1)=1.0, b_{2}(2)=1.4$. The initial data can be assumed to be $x_{1}(0)=0.6, x_{2}(0)=0.4, r_{0}=1$, and we simulate the solution with different values of $\sigma_{i}(k)$.

(i) $\sigma_{1}(1)=0.2, \sigma_{1}(2)=1.0, \sigma_{2}(1)=0.2, \sigma_{2}(2)=0.5$. We have

$$
\begin{aligned}
& \sum_{k \in S} \pi_{k}\left(b_{1}(k)-\frac{\sigma_{1}^{2}(k)}{2}\right)=0.7450>0, \quad \sum_{k \in S} \pi_{k}\left(b_{2}(k)-\frac{\sigma_{2}^{2}(k)}{2}\right) \approx 1.2013>0, \\
& \sum_{k \in S} \pi_{k}\left(\left(b_{1}(k)-\frac{\sigma_{1}^{2}(k)}{2}\right)-\frac{a_{12}}{a_{22}}\left(b_{2}(k)-\frac{\sigma_{2}^{2}(k)}{2}\right)\right) \approx 0.1444>0, \\
& \sum_{k \in S} \pi_{k}\left(\left(b_{2}(k)-\frac{\sigma_{2}^{2}(k)}{2}\right)-\frac{a_{21}}{a_{11}}\left(b_{1}(k)-\frac{\sigma_{1}^{2}(k)}{2}\right)\right) \approx 0.8288>0 .
\end{aligned}
$$

System (62) is persistent in mean, and it is completely consistent by Theorem 3.1. The results are presented clearly in Figures 1 and 2. We draw the stochastic trajectories of $\frac{\log x_{1}(t)}{t}$ and $\frac{\log x_{2}(t)}{t}$ by data simulating. The figures shows that Heun's method for time step $\Delta=2^{-5}$ on $[0,200]$ is superior to others. 


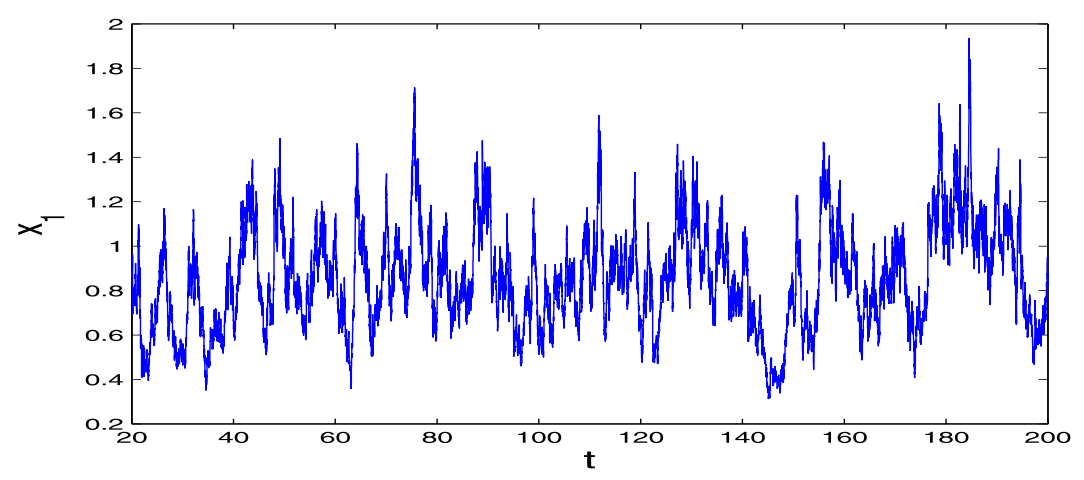

Figure 1 Stochastic trajectory of $x_{1}(t)$ for system $(61)$ with $\sigma_{1}(1)=0.2, \sigma_{1}(2)=1.0, \sigma_{2}(1)=0.2$, $\sigma_{2}(2)=0.5$.

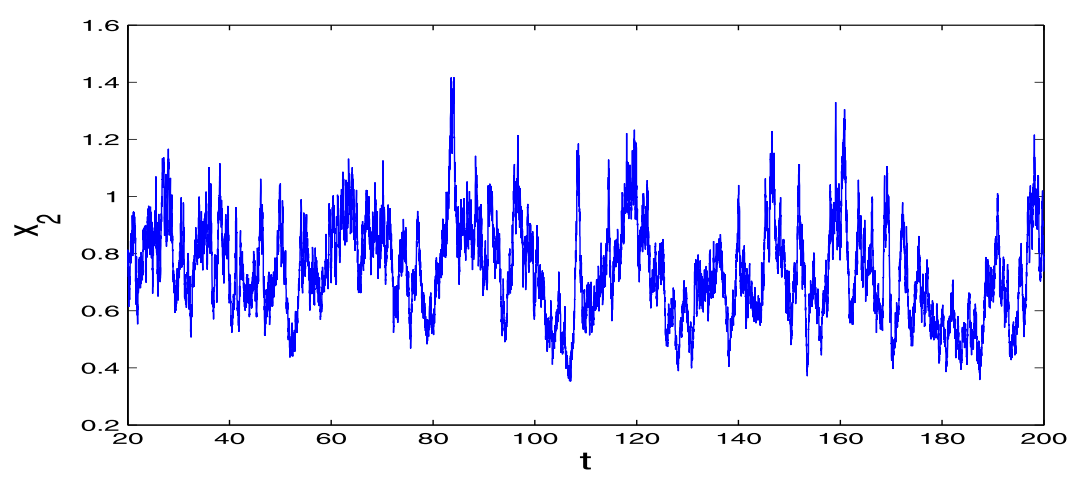

Figure 2 Stochastic trajectory of $x_{2}(t)$ for system (61) with $\sigma_{1}(1)=0.2, \sigma_{1}(2)=1.0, \sigma_{2}(1)=0.2$, $\sigma_{2}(2)=0.5$.

(ii) $\sigma_{1}(1)=\sigma_{1}(2)=2, \sigma_{2}(1)=\sigma_{2}(2)=1.8$. Note that

$$
\sum_{k \in S} \pi_{k}\left(b_{1}(k)-\frac{\sigma_{1}^{2}(k)}{2}\right)=-0.8750<0, \quad \sum_{k \in S} \pi_{k}\left(b_{2}(k)-\frac{\sigma_{2}^{2}(k)}{2}\right)=-0.3200<0 .
$$

Equation (62) is exponentially extinct, and it is completely consistent by Theorem 4.1 for any initial condition $x_{0}>0$. The sample trajectories of $\frac{\log x_{1}(t)}{t}$ and $\frac{\log x_{2}(t)}{t}$ are drawn in Figures 3 and 4 , and they are generated by the Heun scheme for time step $\Delta=2^{-5}$ on $[20,200]$, respectively.

Example 6.2 Consider the three-dimensional stochastic Gilpin-Ayala system with Markovian switching

$$
\left\{\begin{array}{l}
d x_{1}=x_{1}\left(b_{1}(r(t))-0.7 x_{1}^{1.1}-0.4 x_{2}-0.3 x_{3}\right) d t+\sigma_{1}(r(t)) x_{1} d B_{1}(t) \\
d x_{2}=x_{2}\left(b_{2}(r(t))-0.3 x_{1}-x_{2}^{1.5}-0.3 x_{3}\right) d t+\sigma_{2}(r(t)) x_{2} d B_{2}(t) \\
d x_{3}=x_{3}\left(b_{3}(r(t))-0.6 x_{1}-0.4 x_{2}-x_{3}^{1.2}\right) d t+\sigma_{3}(r(t)) x_{3} d B_{3}(t)
\end{array}\right.
$$

where the Markovian chain $r(t)$ and generator $Q$ are defined in the previous example, and $b_{1}(1)=1, b_{1}(2)=1.3, b_{2}(1)=1, b_{2}(2)=1.5, b_{3}(1)=1, b_{3}(2)=0.9, \sigma_{1}(1)=0.6, \sigma_{2}(1)=\sqrt{5}$, 


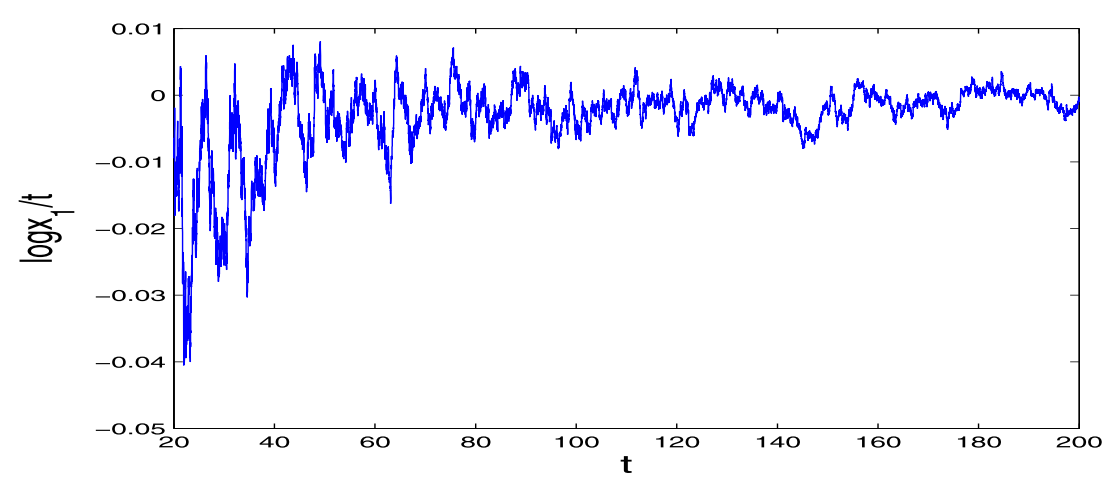

Figure 3 Stochastic trajectory of $\frac{x_{1}(t)}{t}$ for system (61) with $\sigma_{1}(1)=\sigma_{1}(2)=2, \sigma_{2}(1)=\sigma_{2}(2)=1.8$.

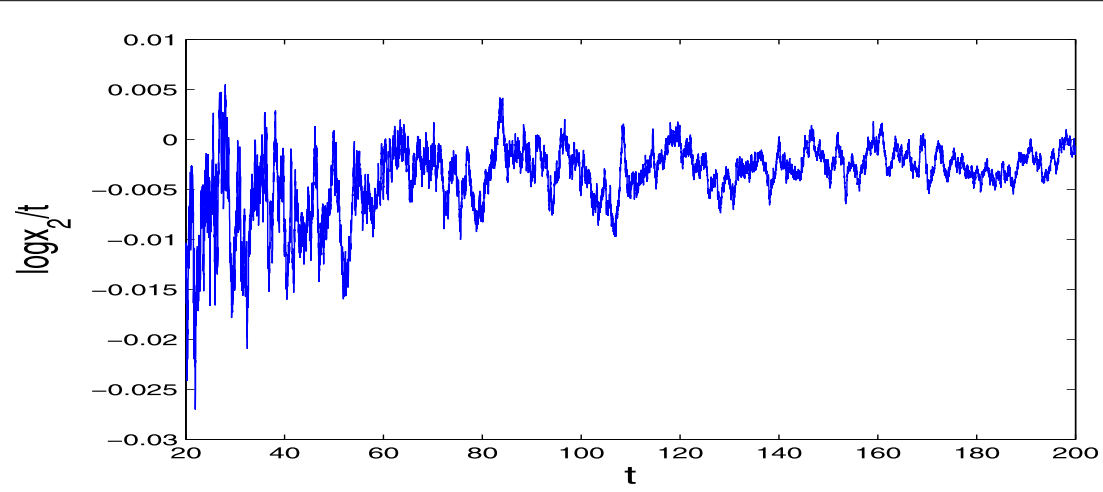

Figure 4 Stochastic trajectory of $\frac{x_{2}(t)}{t}$ for system (61) with $\sigma_{1}(1)=\sigma_{1}(2)=2, \sigma_{2}(1)=\sigma_{2}(2)=1.8$.

$\sigma_{3}(1)=2, \sigma_{1}(2)=0.4, \sigma_{2}(2)=\sqrt{2}, \sigma_{3}(2)=2$. The initial data $x_{1}(0)=0.4$ and $x_{2}(0)=0.6$ are given. We can show that the conditions of Theorem 5.1 are met exactly. A simple computation yields

$$
\begin{aligned}
& \sum_{k \in S} \pi_{k}\left(b_{1}(k)-\frac{\sigma_{1}^{2}(k)}{2}\right)=1.12>0, \quad \sum_{k \in S} \pi_{k}\left(b_{2}(k)-\frac{\sigma_{2}^{2}(k)}{2}\right)=0, \\
& \sum_{k \in S} \pi_{k}\left(b_{3}(k)-\frac{\sigma_{3}^{2}(k)}{2}\right)=-1.075<0, \\
& a(11)-a(21)=0.4>0, \quad a(22)-a(12)=0.6>0, \\
& \limsup _{t \rightarrow \infty} \frac{1}{t} \int_{0}^{t} x_{1}^{\theta_{1}}(s) d s=1.60, \quad \quad \limsup _{t \rightarrow \infty} \frac{\log x_{2}(t)}{t} \leq 0, \\
& \limsup _{t \rightarrow \infty} \frac{\log x_{3}(t)}{t} \leq-1.075 .
\end{aligned}
$$

The species 1 is persistent in mean, and species 2 and 3 are exponentially extinct by Theorem 5.1. The system is tested in detail by the Heun method in Figures 5, 6, and 7, and the data simulating results validate the correctness and effectiveness of the proposed methods. 


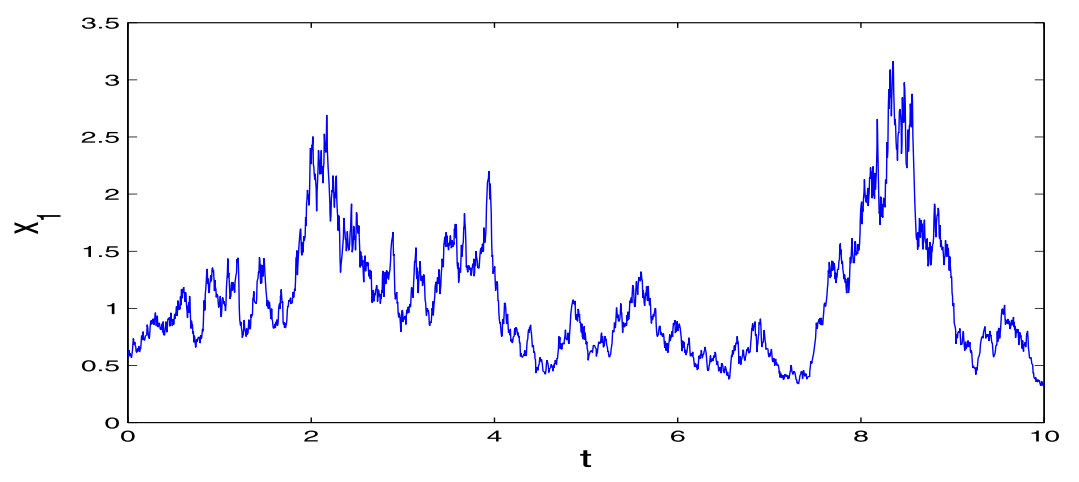

Figure 5 Stochastic trajectory of $x_{1}(t)$ for system (62).

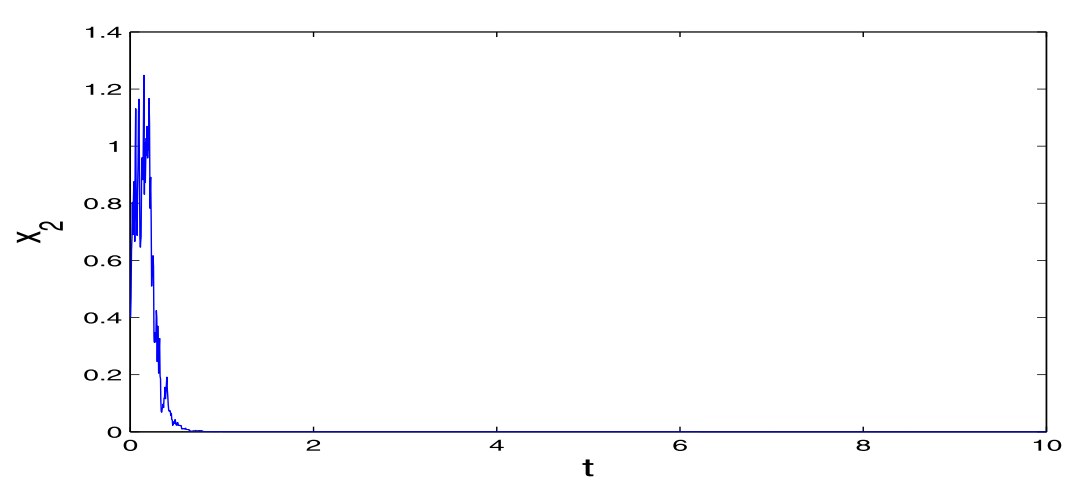

Figure 6 Stochastic trajectory of $x_{2}(t)$ for system (62).

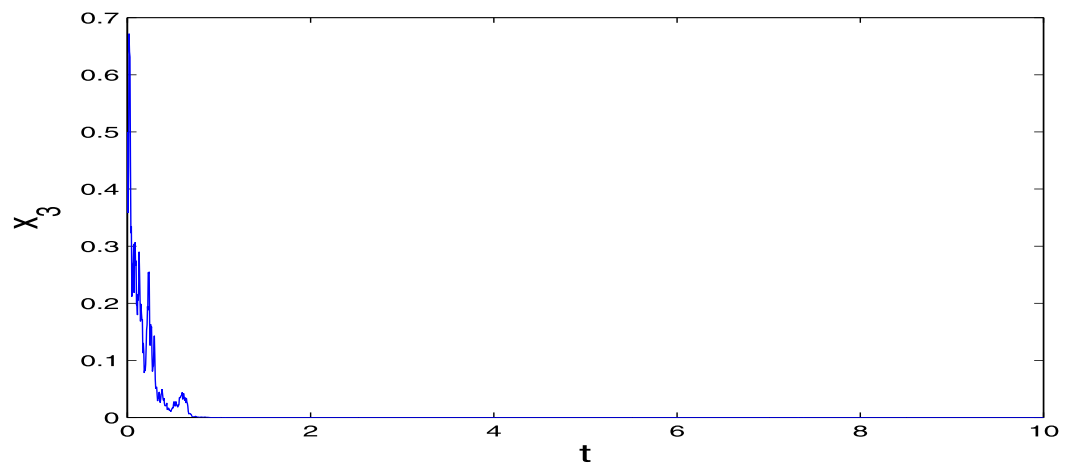

Figure 7 Stochastic trajectory of $x_{3}(t)$ for system (62).

\section{Conclusions}

The asymptotic behavior for stochastic competitive Gilpin-Ayala systems with regime switching has been investigated. Firstly, generalized criteria on persistence in mean are established by utilizing stochastic comparison principle and novel analysis techniques. Secondly, sufficient criteria on extinction are obtained by the space-decomposition method and the stochastic LaSalle theorem. Thirdly, sufficient conditions for partial persistence in mean and partial extinction are derived based on the criteria and a novel Lyapunov func- 
tion. Two numerical examples are provided to illustrate the superiority and effectiveness of the proposed approaches.

\begin{abstract}
Acknowledgements
The research work is supported by the National Science Foundation of China (Grant Nos. 61304070, 11671118, 61374080), the China Postdoctoral Science Foundation funded project (2016M601698), the Fundamental Research Funds for the central Universities of China (Grant No. 2015B19814) and the Alexander von Humboldt Foundation of Germany (Fellowship CHN/1163390), Qing Lan Project of Jiangsu Province, the Natural Science Foundation of Jiangsu Province (BK20161552). The first author would like to thank professor Jifeng Chu for his support and guidance. Without him this work would not be possible.
\end{abstract}

\title{
Competing interests
}

The authors declare that they have no competing interests.

\section{Authors' contributions}

The authors have made the same contribution. All authors read and approved the final manuscript.

\section{Author details}

${ }^{1}$ College of Science, Hohai University, Nanjing, Jiangsu 210098, China. ${ }^{2}$ School of Mathematics, Southeast University, Nanjing, Jiangsu 210096, China. ${ }^{3}$ School of Mathematical Sciences and Institute of Finance and Statistics, Nanjing Normal University, Nanjing, Jiangsu 210023, China.

\section{Publisher's Note}

Springer Nature remains neutral with regard to jurisdictional claims in published maps and institutional affiliations.

Received: 23 September 2017 Accepted: 7 December 2017 Published online: 21 December 2017

\section{References}

1. Bao, J, Mao, X, Yin, G, Yuan, C: Competitive Lotka-Volterra population dynamics with jumps. Nonlinear Anal. 74, 6601-6616 (2011)

2. Khasminskii, RZ: Stochastic Stability of Differential Equations. Springer, Berlin (2012)

3. Khasminskii, RZ, Zhu, C, Yin, G: Stability of regime switching diffusions. Stoch. Process. Appl. 117, 1037-1051 (2007)

4. Li, X, Mao, X: Population dynamical behavior of non-autonomous Lotka-Volterra competitive system with random perturbation. Discrete Contin. Dyn. Syst. 24, 523-593 (2009)

5. Jiang, D, Ji, C, Li, X, O'Regan, D: Analysis of autonomous Lotka-Volterra competition systems with random perturbation. J. Math. Anal. Appl. 390, 582-595 (2012)

6. Li, X, Jiang, D, Mao, X: Population dynamical behavior of Lotka-Volterra system under regime switching I. J. Comput. Appl. Math. 232, 427-448 (2009)

7. Li, X, Gray, A, Jiang, D, Mao, X: Sufficient and necessary conditions of stochastic permanence and extinction for stochastic logistic populations under regime switching. J. Math. Anal. Appl. 376, 11-28 (2011)

8. Liu, L, Shen, Y: New criteria on persistence in mean and extinction for stochastic competitive Lotka-Volterra systems with regime switching. J. Math. Anal. Appl. 430, 306-323 (2015)

9. Mao, X, Marion, G, Renshaw, E: Environmental noise suppresses explosion in population dynamics. Stoch. Process. Appl. 97, 95-110 (2002)

10. Mao, X, Yuan, C: Stochastic Differential Equations with Markovian Switching. Imperial College Press, London (2006)

11. Zhu, C, Yin, G: Asymptotic properties of hybrid diffusion systems. SIAM J. Control Optim. 46, 1155-1179 (2007)

12. Bountis, T, Vanhaecke, P: Lotka-Volterra systems satisfying a strong Painlevé property. Phys. Lett. A 380, 3977-3982 (2016)

13. Mao, X, Marion, G, Renshaw, E: Asymptotic behavior of the stochastic Lotka-Volterra model. J. Math. Anal. Appl. 287, $141-156(2003)$

14. Tian, G, Zhang, G: Stability of traveling wavefronts for a discrete diffusive Lotka-Volterra competition system. J. Math. Anal. Appl. 447, 222-242 (2017)

15. Zhu, C, Yin, G: On competitive Lotka-Volterra model in random environments. J. Math. Anal. Appl. 357, 154-170 (2009)

16. Wang, K, Zhu, Y, Zhu, H: New results on the stochastic Gilpin-Ayala model with delays. Filomat 6, 1431-1440 (2016)

17. Wu, R, Zou, X, Wang, K: Asymptotic properties of stochastic hybrid Gilpin-Ayala system with jumps. Appl. Math. Comput. 249, 53-66 (2014)

18. Liu, L, Zhu, Q: Analysis of stochastic Gilpin-Ayala competition system. Math. Probl. Eng. 2014, 1-10 (2014)

19. Settati, A, Lahrouz, A: On stochastic Gilpin-Ayala population model with Markovian switching. Biosystems 130, 17-27 (2015)

20. Mao, X: Stochastic Differential Equations and Applications, 2nd edn. Horwood, Chichester (2007)

21. Watanabe, S, Ikeda, N: Stochastic differential equations and diffusion processes. North Holland, Amsterdam (1981)

22. Mao, X: A note on the LaSalle-type theorems for stochastic differential delay equations. J. Math. Anal. Appl. 268, 125-142 (2002) 